ARTICLE

https://doi.org/10.1038/s41467-019-10918-2

\title{
Structure of the substrate-engaged SecA-SecY protein translocation machine
}

Chengying $M a^{1,4}$, Xiaofei $\mathrm{Wu}^{1,4}$, Dongjie Sun ${ }^{1}$, Eunyong Park ${ }^{2}$, Marco A. Catipovic ${ }^{3}$, Tom A. Rapoport (D) ${ }^{3}$, Ning Gao ${ }^{1} \&$ Long Li ${ }^{1}{ }^{1}$

The Sec61/SecY channel allows the translocation of many proteins across the eukaryotic endoplasmic reticulum membrane or the prokaryotic plasma membrane. In bacteria, most secretory proteins are transported post-translationally through the SecY channel by the SecA ATPase. How a polypeptide is moved through the SecA-SecY complex is poorly understood, as structural information is lacking. Here, we report an electron cryo-microscopy (cryo-EM) structure of a translocating SecA-SecY complex in a lipid environment. The translocating polypeptide chain can be traced through both SecA and SecY. In the captured transition state of ATP hydrolysis, SecA's two-helix finger is close to the polypeptide, while SecA's clamp interacts with the polypeptide in a sequence-independent manner by inducing a short $\beta$ strand. Taking into account previous biochemical and biophysical data, our structure is consistent with a model in which the two-helix finger and clamp cooperate during the ATPase cycle to move a polypeptide through the channel.

\footnotetext{
${ }^{1}$ State Key Laboratory of Membrane Biology, Peking-Tsinghua Center for Life Sciences, School of Life Sciences, Peking University, Beijing, China. ${ }^{2}$ University of California-Berkeley, Stanley Hall, Berkeley, CA 94720, USA. ${ }^{3}$ Department of Cell Biology, Howard Hughes Medical Institute and Harvard Medical School, 240 Longwood Avenue, Boston, MA 02115, USA. ${ }^{4}$ These authors contributed equally: Chengying Ma, Xiaofei Wu. Correspondence and requests for materials should be addressed to T.A.R. (email: tom_rapoport@hms.harvard.edu) or to N.G. (email: gaon@pku.edu.cn) or to L.L. (email: long_li@pku.edu.cn)
} 
$\mathrm{P}$ rotein translocation is a universal and essential process that allows the export of secretory proteins from cells and the integration of membrane proteins into lipid bilayers ${ }^{1-6}$. The central translocation component is an evolutionarily conserved protein-conducting channel, the Sec61 channel in eukaryotes and the SecY channel in prokaryotes. Both are formed from a heterotrimeric protein complex, containing a large subunit with ten trans-membrane (TM) helices (Sec61a in eukaryotes and SecY in prokaryotes), and two small subunits that in most species contain only one TM segment (called Sec61 $\beta$ and Sec61 $\gamma$ in eukaryotes and SecG and SecE in bacteria). The Sec61 $\beta /$ SecG subunit is not essential for the function of the channel ${ }^{7}$. The Sec61/SecY channel has an hourglass shape, with an empty cytosolic funnel and an extracellular funnel that is filled with a plug domain ${ }^{8-11}$. A constriction is formed in the middle of the channel by a ring of conserved hydrophobic amino acids, called the pore ring. During translocation, the plug is displaced, and the polypeptide chain moves through the pore ring across the membrane. A lateral gate in Sec61a/SecY allows hydrophobic signal sequences of secretory proteins or TM segments of membrane proteins to exit into the lipid phase.

The channel needs to associate with a partner that provides the driving force for translocation (for review, see ref. ${ }^{5}$ ). In cotranslational translocation, the $\mathrm{Sec} 61 / \mathrm{SecY}$ channel associates with the translating ribosome, such that a nascent polypeptide is moved from the ribosome tunnel into the membrane channel. In posttranslational translocation in eukaryotes, the Sec61 channel partners with another membrane protein complex, the Sec62/63 complex, as well as with the luminal chaperone BiP, a member of the Hsp70 family of ATPases. BiP binds to the polypeptide as it emerges into the ER lumen and prevents it from sliding back into the cytosol ${ }^{12}$. In posttranslational translocation in bacteria, the SecY channel associates with the SecA ATPase. SecA uses the energy of ATP hydrolysis to move polypeptides through the channel, but the mechanism of translocation is poorly understood.

SecA has two nucleotide-binding domains (NBD1 and NBD2), which bind the nucleotide at their interface ${ }^{13}$. A two-helix finger (THF), consisting of two helices connected by a loop, inserts into the cytoplasmic funnel of the SecY channel ${ }^{14,15}$. A clamp, formed by rotation of the polypeptide-cross-linking domain (PPXD) toward NBD2, positions the translocating polypeptide chain above the channel ${ }^{16}$.

Several mechanisms have been proposed for SecA. In a ratcheting model ${ }^{17,18}$, SecA's THF senses bulky amino acid residues of a substrate. When it encounters such a residue, SecA converts from the ADP-bound to the ATP-bound state and the SecY channel opens, allowing the residue to move through the pore. Following ATP hydrolysis, the channel closes, trapping the bulky residue on the other side of the pore ring. In a powerstroke model ${ }^{19}$, ATP binding of SecA causes the THF to move toward the channel and push the polypeptide chain into the channel; following ATP hydrolysis, the finger retracts and allows sliding of the chain in either direction. During retraction of the THF, the clamp holds the polypeptide chain, so that the chain is not dragged backward when the THF resets ${ }^{14,20}$. In the originally proposed version of a power-stroke model, large domains of SecA reach entirely through membrane to deliver the substrate to the other side si,22 $^{21}$

An understanding of the mechanism of posttranslational protein translocation requires structures of the active Sec61/SecY channel and visualization of the translocating polypeptide substrate. Cross-linking experiments provided evidence that a substrate contacts both the clamp and the THF ${ }^{16}$, but the exact path of the translocating chain could not be deduced. A previously determined crystal structure contained the detergent-solubilized
SecY channel, the SecA ATPase, and a segment of a secretory protein (proOmpA) fused into the THF of SecA (SecA-OAins) ${ }^{23}$ The substrate segment inserted as a loop into the channel, with the signal sequence forming a helix outside the lateral gate. However, the construct design did not allow determination of the polypeptide path through SecA, and it is even unclear to what extent the artificial nature of the construct affected the position of the substrate in SecY. Here, we report a structure of an active translocon with a translocating polypeptide chain caught in the act of moving through SecA and SecY.

\section{Results}

Assembly of an active translocation complex. To obtain a structure of a translocating SecA-SecY-substrate complex, we assembled in E. coli cells a complex containing Bacillus subtilis SecA (residues 1-778), Geobacillus thermodenitrificans SecYE, and a translocating polypeptide substrate. SecA lacks C-terminal residues, which are dispensable for its function ${ }^{24}$. The substrate consists of the signal sequence of proOmpA, a linker polypeptide segment, and superfolder Green Fluorescence Protein (sfGFP) 25 (Fig. 1a, b). The bulky sfGFP moiety prevents the C-terminus of the substrate from moving through the SecA-SecY complex, thus generating a translocation intermediate. To reduce the flexibility of the GFP moiety, an anti-GFP nanobody (enhancer) ${ }^{26}$ was fused to the C-terminus of SecA. Stabilization of the channelinserted polypeptide was achieved by a disulfide bridge formed without addition of an exogenous oxidant; it linked a cysteine introduced C-terminally of the signal sequence to a cysteine placed into the plug domain of SecY (Fig. 1a, b). The complex was purified in the presence of ADP.BeFx to lock SecA in the transition state of ATP hydrolysis. The complex was then reconstituted into nanodiscs ${ }^{27}$, generating a physiological lipid environment for the channel (Supplementary Fig. 1). Before analysis by cryo-EM, an anti-SecY nanobody was added to reduce the flexibility of the channel on its periplasmic side ${ }^{23}$. Stabilization agents such as nanobodies and disulfide cross-links proved crucial to maintain the translocation complex for crystallographic studies, though it is unclear whether they improved the cryo-EM map quality. A cryo-EM density map was obtained with an overall resolution of $3.5 \AA$ (Fig. 1c, Supplementary Fig. 2, and Supplementary Table 1). The obtained map was of sufficient quality to build atomic models for SecA and SecYE (Fig. 1d), including segments that were poorly resolved in previous structures $^{14,23}$, such as the loop between TM6 and TM7 of SecY (Supplementary Fig. 3). Most of the polypeptide substrate could also be traced (Fig. 1d).

SecA and SecY in the active translocation complex. In the active translocation complex, the overall conformations of SecA and SecY are similar to those in previous structures ${ }^{14,23}$. SecA binds to several cytosolic loops of SecY, including the loop between TM8 and TM9 (L8/9), which interacts with the PPXD, and the Cterminal tail, which binds through the conserved hydrophobic residues Y425 and F428 into a groove in the helical scaffold domain (HSD) (Fig. 2a, b, Supplementary Fig. 3g). The loop between TM6 and TM7 of SecY (L6/7) has a previously unrecognized role in SecA interaction. It is surrounded by L8/9 of SecY, and by the THF and PPXD of SecA (Fig. 2a). Both SecY loops together help to induce a closed conformation of SecA's clamp. SecA also interacts with the lipid surface of the nanodiscs. It binds through two charged regions (Fig. 1c), one including residues R553, R576, and K583 in the HSD, and the other including residues R645 and E646 in the helical wing domain (HWD) (Supplementary Fig. 4a and b). The N-terminal 13 amino acids of 
a

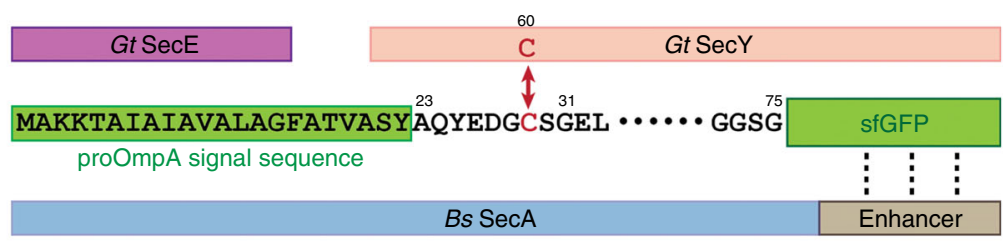

b

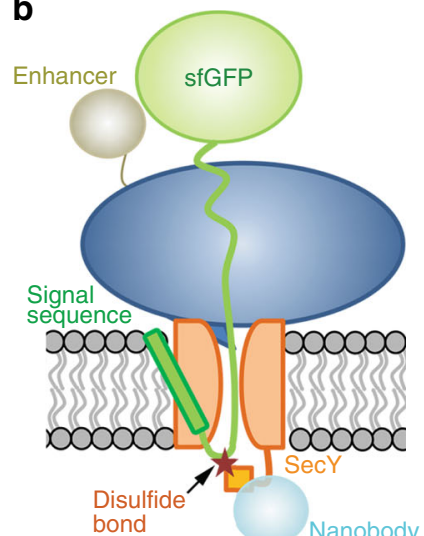

C
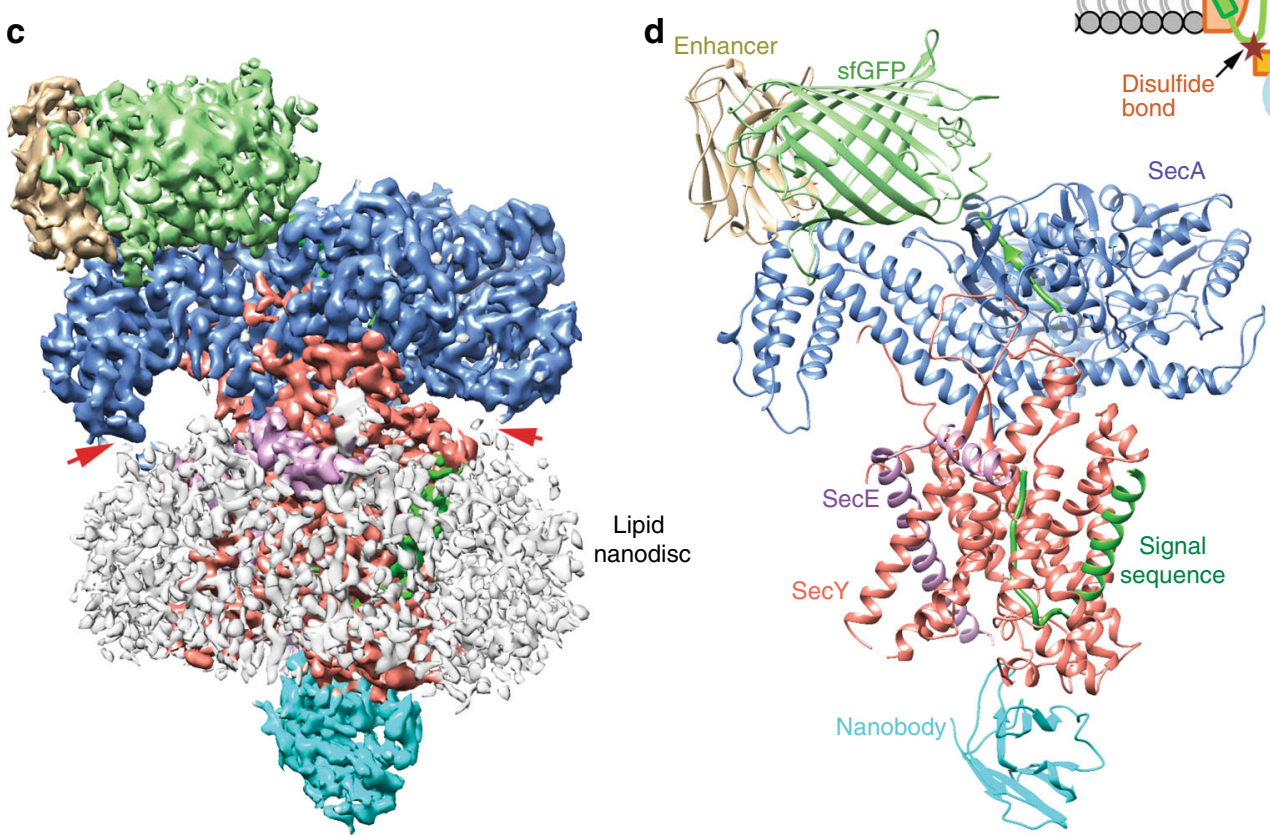

Fig. 1 Overall structure of the SecA-SecY-substrate complex a Components of the translocation complex. The protein sequences are shown schematically as bars in different colors. G. thermodenitrificans (Gt) SecE, purple; SecY, salmon; sfGFP, green; B. subtilis (Bs) SecA, blue; and Enhancer, tan. The proOmpA signal sequence is highlighted in green. Cysteines (C) used for cross-linking (double arrow) are shown in red. The interaction between sfGFP and Enhancer are indicated by three dashed lines. The positions of some residues are indicated. $\mathbf{b}$ Scheme of the assembled translocation complex. c Cryo-EM density map of the SecA-SecY-substrate complex, with density for the individual proteins colored as in (a) and (b). The lipid nanodisc is colored in gray. Interaction sites between SecA and the lipid surface are indicated by red arrowheads. $\mathbf{d}$ Ribbon model of the complex. Individual proteins are colored and labeled as in (a) and (b)

SecA, implicated for E. coli SecA in lipid binding ${ }^{19,28}$, are invisible and thus likely flexible.

The plug in the SecY channel occupies a more central position than in the SecA-OAins/SecY crystal structure and adopts a distorted helical conformation, rather than forming the previously observed $\beta$-strands (Fig. 2c, d). Although the cause for the conformational change is unclear, the plug can adopt different structures in different species and reforms from neighboring segments when deleted ${ }^{29}$. It thus appears that the only important function of the plug is to seal the channel in its closed state. The $\mathrm{Sec} \mathrm{Y}$ channel is surrounded by lipids from the nanodisc (Fig. 1c). Some phospholipid molecules seem to be bound specifically, including one at the back of the channel (Supplementary Figs 5a and $b$ ), which is positioned parallel to the membrane surface with the hydrophilic head group deep inside the extracellular funnel. It might thus interact with the translocating polypeptide chain, although its functional significance remains unclear.

The polypeptide substrate in the SecY channel. The structure shows how the polypeptide substrate is translocated through the SecA-SecY complex (Fig. 3a, b). The N-terminal signal sequence forms a helix that is tilted by $\sim 45^{\circ}$ with respect to the membrane plane and bound to a groove on the outside of the lateral gate of the SecY channel, as in the SecA-OAins/SecY crystal structure $^{23}$. However, the helix is shifted by half a turn toward the cytosolic side of the membrane (Fig. 3c). To accommodate this change, TMs 7 and 8 of SecY are tilted by $\sim 10^{\circ}$ toward the cytosolic side and the extracellular ends of TMs 7 and 3 approach each other, closing the lateral gate on the periplasmic side. These differences indicate that the signal sequence binding site might be flexible or was somewhat distorted in the previous artificial construct. Although bound to a groove of the SecY channel, the hydrophobic part of the signal sequence is exposed to the lipids of the nanodisc, supporting the idea that it is recognized mainly by partitioning into the lipid phase. This is consistent with the ability of signal sequences to be cross-linked to lipids ${ }^{30,31}$ and with the correlation between the partitioning of synthetic peptides into hydrophobic solvents and their function as signal sequences in vivo ${ }^{32}$. Lipid partitioning explains why signal sequences can vary in length and sequence, but require a hydrophobic peptide segment ${ }^{23}$. Some lipids seem to bind to certain sites at the interface to SecY (Supplementary Fig. 5c), perhaps participating in the interaction between the signal sequence and channel. 
a

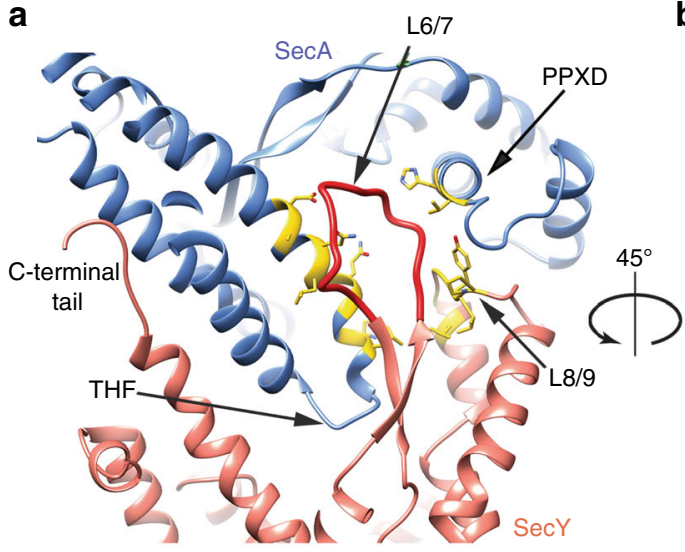

C

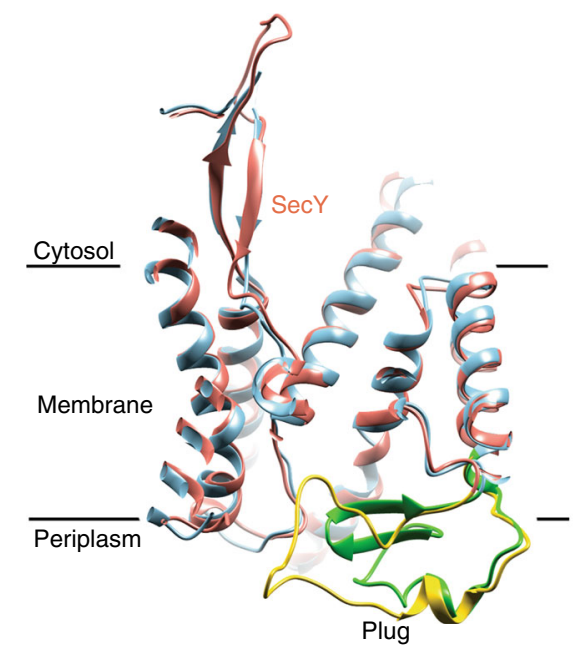

b

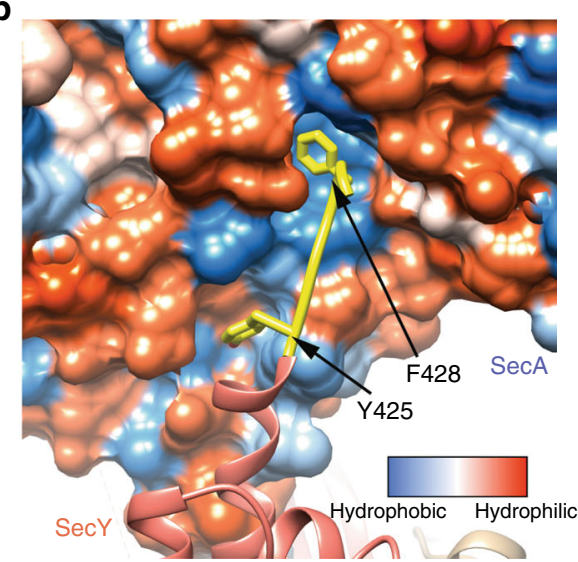

d

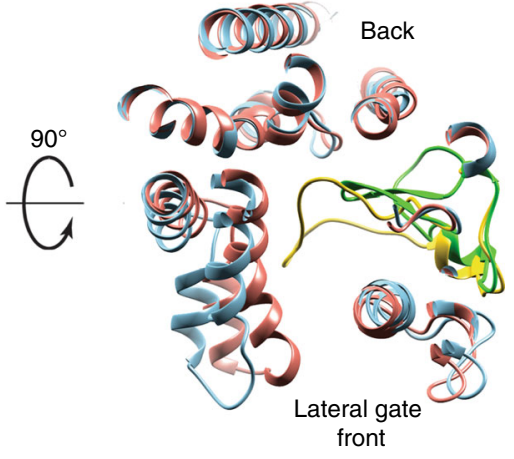

Fig. 2 The interaction between SecA and SecY and changes in SecY's plug domain. a Interaction of the loop between TMs 6 and 7 of SecY (L6/7) (red) with the PPXD (blue), the THF, and the loop between TMs 8 and 9 (L8/9). The regions involved are colored yellow. b Interaction of the C-terminal tail (yellow) of SecY with SecA. SecA is shown as a space-filling model with residues colored according to their hydrophobicity. Interacting SecY residues are shown as yellow sticks. c Comparison between the plug regions in the cryo-EM structure and the crystal structure of SecA-OAins/SecY (PDB ID: 5EUL). The SecY molecule and plug are colored salmon and yellow, respectively, for the cryo-EM structure, and cyan and green, respectively, for the crystal structure. $\mathbf{d}$ Top view of (c)

The polypeptide segment following the signal sequence makes a U-turn in the extracellular funnel. The loop is significantly shorter than in the crystal structure ( 9 versus 19 residues, counting from the first residue after the signal sequence, Ala23, to the residue in the pore ring (Fig. 3d). The shorter extracellular loop could correspond to an earlier stage of translocation, in which fewer residues of the C-terminal part of the loop have moved across the membrane. In both structures, a glycine residue is trapped in the pore ring (Gly31 and Gly41, in the present and previous structures, respectively). The pore ring residues of SecY form a gasket-like seal around these glycine residues (Supplementary Fig. 5d), preventing even small molecules, such as ions, to permeate through the membrane during translocation ${ }^{33}$. Glycines are probably trapped in the structures because they minimize pore expansion in the presence of a translocating polypeptide and thus lead to a more stable state. The polypeptide adopts an extended conformation inside the SecY channel. The polypeptide chain is almost perpendicular to the plane of the lipid bilayer and extends from the loop in the extracellular space to the cytoplasmic side of the membrane (Fig. 3b, d).

The polypeptide substrate in SecA. The polypeptide chain could be traced all the way from SecY back to its entry point into SecA.
The least resolved region is a small segment at the SecY-facing end of SecA's clamp (encircled in Fig. 3b), where density for the polypeptide was only visible at lower thresholds, suggesting that this segment is flexible when it passes through. The connection between the polypeptide substrate and the C-terminal sfGFP is also flexible, although both the sfGFP moiety and the interacting SecA-fused nanobody are visible in the structure (Fig. 1c).

At its entry point into SecA, the polypeptide chain is embraced by the clamp, formed from segments of the PPXD and HSD (Fig. 4a). Contrary to a previous prediction ${ }^{14}$, the NBD2 does not contact the polypeptide. On one side of the polypeptide path, a loop of the PPXD (residues 314-325) reaches into a cavity between NBD1 and NBD2 (Fig. 4a) and contains the highly conserved Arg367 residue (numbering for B. subtilis SecA; Fig. 4a), the mutation of which impairs translocation in E. coli ${ }^{34}$. On the other side of the path, in the back of the clamp, are two $\beta$ strands that connect NBD1 with the PPXD. Both the substrate and a segment right after the PPXD loop (residues 326-331) adopt short $\beta$-strands, so that the four strands together form a $\beta$ sheet (Fig. 4a, b, Supplementary Fig. 3i and j). The $\beta$-strand in PPXD is caused by the presence of substrate, as it is not seen in previous structures, including those of E. coli and M. tuberculosis SecA ${ }^{13,35,36}$. Similarly, the $\beta$-strand in the substrate itself is also 
a

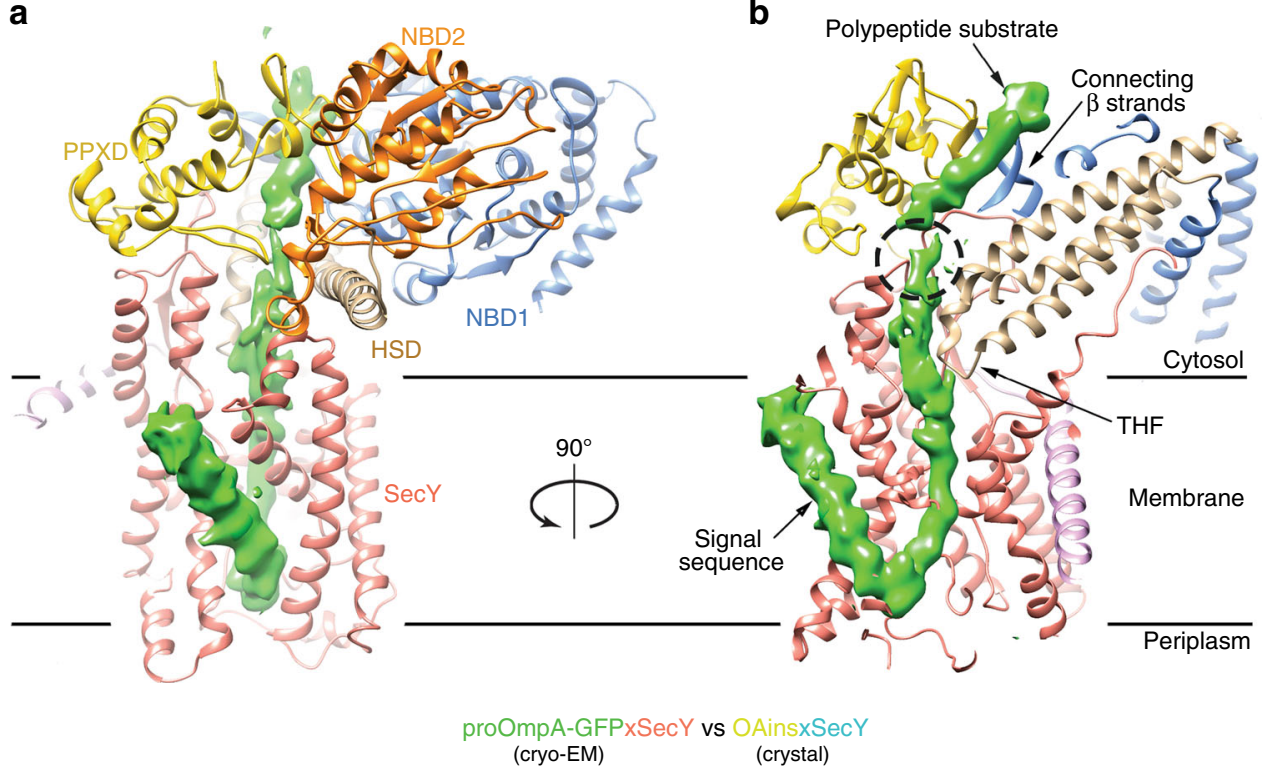

C

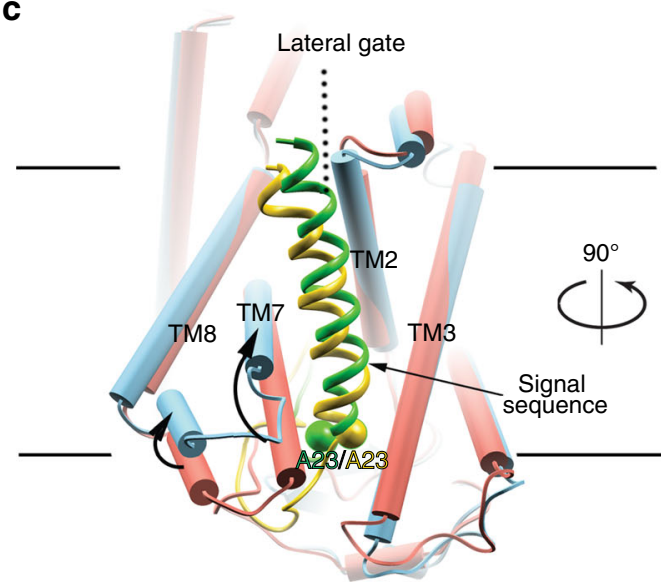

d b

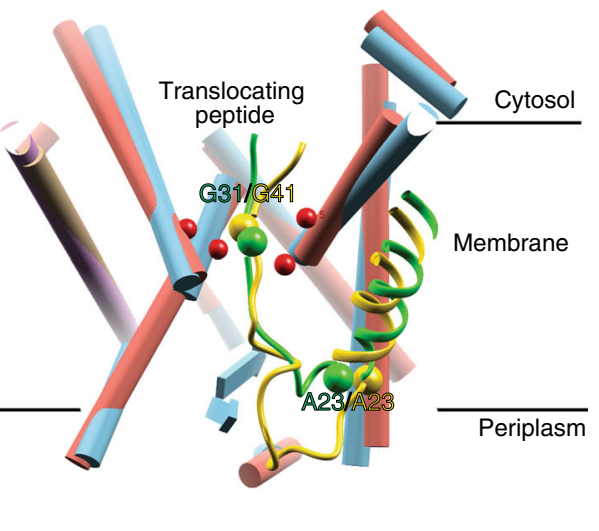

Fig. 3 The translocating polypeptide in the SecA-SecY complex. a Density for the polypeptide (green) is shown in a ribbon diagram of the translocation complex. SecA domains are shown in different colors. b As in (a), but a cut-away view from a different angle. The dashed circle indicates a region where the substrate could not be traced, likely because it is flexible. c Comparison of the signal sequences and lateral gates between the cryo-EM and crystal (PDB ID: 5EUL) structures. SecY is shown as cylinders in salmon and cyan, respectively. The signal sequences are shown as ribbon diagrams, colored green and yellow for the cryo-EM and crystal structures, respectively. The movements of TM7 and TM8 are indicated by arrows. $\mathbf{d}$ As in (c), but cut-away side view from a different angle. The first residue after the signal sequence (Ala23) and the Gly residues trapped inside the pore rings (red balls) are labeled and shown as balls

induced. In fact, crystal structures of SecA in isolation showed that the normally unstructured C-terminus of $\operatorname{SecA}^{13}$ or an $\alpha$ helix from a neighboring SecA molecule ${ }^{37}$ are induced to form $\beta$ strands that augment the two connecting $\beta$-strands. A small peptide also binds to the same site in a C-terminally truncated SecA construct ${ }^{38}$. The $\beta$-sheets in all these structures are superimposable although they contain different amino acid sequences (Fig. $4 \mathrm{~d}$ ), as expected for $\beta$-strands interacting by $\mathrm{H}$ bonding of the polypeptide backbones.

The substrate segment closest to the THF shows some variability in its conformation, suggesting that it does not strongly interact with the THF; the polypeptide has a rather fragmented density in this region and it occupies slightly different positions in different $3 \mathrm{D}$ classes (Supplementary Fig. 6). The THF itself has a similar position as in the SecA-SecYEG crystal structure $^{14}$ (Fig. 5a). Several residues of the THF (H739, Y743, and Q745) are in the vicinity of the polypeptide (Fig. 5b; Supplementary Fig. 3h), but they do not make strong contacts. Together with cytosolic loops of SecY, the THF guides the polypeptide chain into the channel (Figs. 3b, 5c). The SecG subunit of the SecY complex, omitted in our structure, may also play a role, as its cytosolic domain blocks the SecY channel in its idle state ${ }^{10}$ and would be close to the polypeptide substrate in the active channel (Fig. 5d, e).

\section{Discussion}

Our cryo-EM structure shows the path of a translocating polypeptide chain from its entry point into the SecA ATPase all the way to the extracellular side of the SecY channel. The structure therefore approximates better a translocating state of the polypeptide chain than a previous crystal structure, in which a substrate segment was fused into the THF of $\operatorname{Sec}^{20}$. Nevertheless, it is possible that some distortion of the truly translocating state was caused in our structure by the use of nanobodies or disulfide bridge cross-linking. Cryo-EM structures have also been reported for active, ribosome-associated mammalian Sec61 channels, but the translocating polypeptide chains were invisible inside the channel ${ }^{39-41}$. 
a

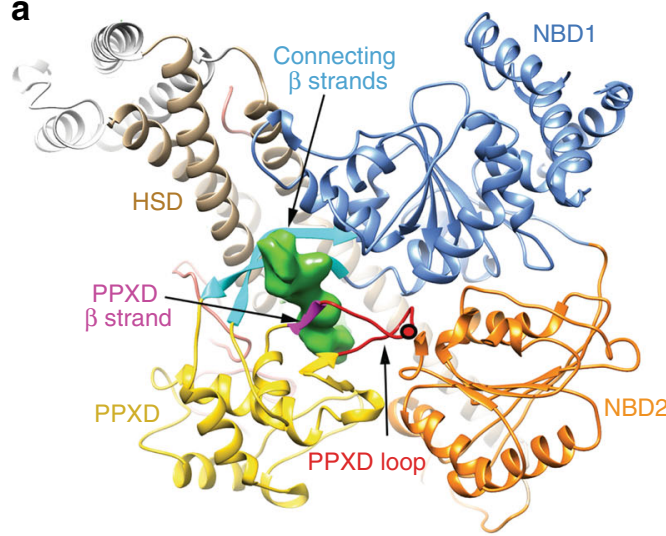

d

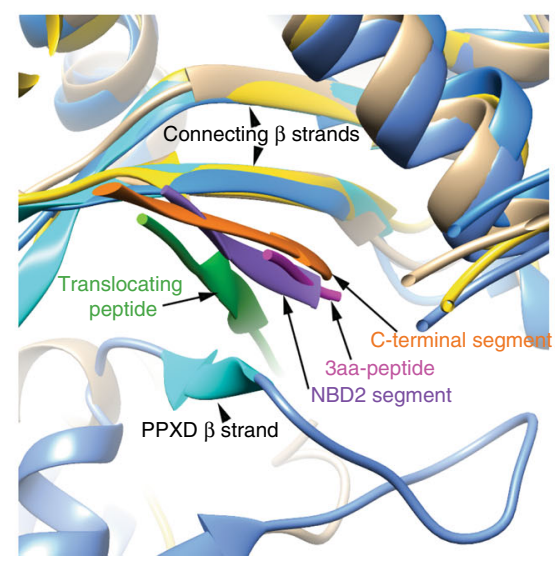

b
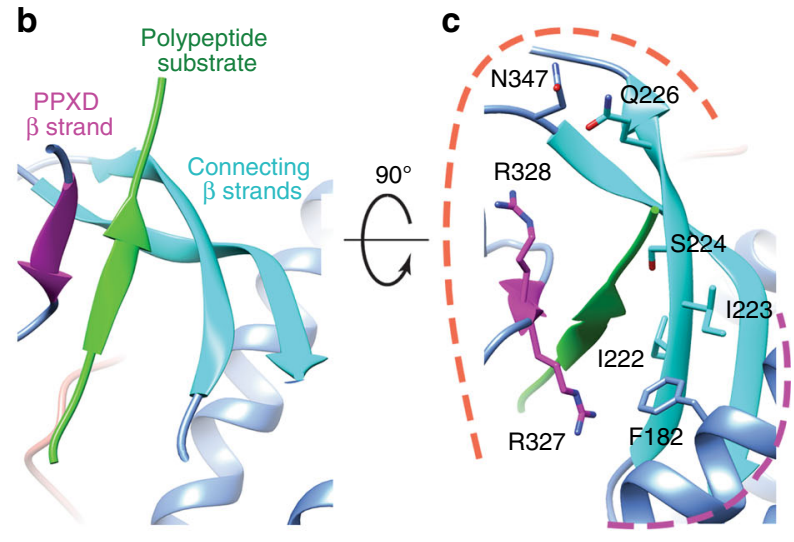

Fig. 4 Polypeptide substrate in SecA. a Top view of the SecA-SecY-substrate complex, showing polypeptide entry into SecA. The SecY channel is underneath SecA and not shown. Substrate density is shown in green. The conserved Arg367 in the PPXD loop is indicated as a red dot. $\mathbf{b}$ Close-up view of the $\beta$-sheet formed by substrate binding. The connecting $\beta$-strands at the back of the clamp are in shown in cyan, the PPXD $\beta$-strand in magenta, and the $\beta$ strand formed by the substrate in green. c SecA residues surrounding the polypeptide substrate are shown as sticks. Hydrophilic and hydrophobic residues, clustered on different sides, are highlighted with orange and purple dashed curves. $\mathbf{d}$ Interaction of the connecting $\beta$ strands with different peptides. Shown is a superposition of different structures, in which $\beta$-strands were induced. The alignment is based on the connecting $\beta$-strands. The peptides are derived from (1) the translocating peptide in the present structure (green); (2) a C-terminal SecA segment (orange, PDB 1M74); (3) three amino acids of a synthetic peptide (red, PDB 3JV2); and (4) a segment of NBD2 of a neighboring SecA molecule in the crystal (purple, PDB 2IBM)

Our cryo-EM structure and previous crystal structures ${ }^{13,37,38}$ indicate that SecA induces the polypeptide substrate to form a short $\beta$-strand that augments a $\beta$-sheet at the back of the clamp. Binding is independent of the specific amino acid sequence, as it involves only the polypeptide backbone, a conclusion that is supported by the fact that several different peptides are induced to form $\beta$-strands (Fig. 4d). This interaction would allow any substrate segment entering SecA to bind, which may contribute to the ability of SecA to translocate a broad range of diverse polypeptides. However, some amino acid side chains of SecA's clamp seem to interact with the polypeptide chain (e.g., R327, F182, and S224; Fig. 4c), which suggests that there may be some preference of the clamp for certain substrate sequences ${ }^{19,42-44}$.

Our structure cannot discriminate between the power-stroke and ratcheting models for SecA function. However, recent singlemolecule FRET experiments support a power-stroke model ${ }^{20}$, as the THF is closest to the SecY channel when SecA is in the ATPbound state, at an intermediate position in the transition state of ATP hydrolysis (ADP.BeFx), and farthest away in the ADPbound state. These data imply that the actual power-stroke occurs during ATP binding to SecA, when the THF tip interacts with the polypeptide and pushes it into the SecY channel.

In our structure, SecA is in the transition state of ATP hydrolysis (ADP.BeFx). Consistent with the single-molecule FRET data, which indicate that in this nucleotide state the
THF should be retracting from the channel, we observe the THF in proximity of the translocating polypeptide chain, but not strongly interacting with it (Fig. 5b). Biochemical data implicate residue Y743 at the fingertip in substrate movement ${ }^{15,19}$, and in our structure this residue is still close to the polypeptide. The single-molecule FRET data indicate that the clamp is closed while the THF retracts, and this again is consistent with our structure, in which the clamp embraces the polypeptide (Fig. 4). The closure of the clamp during ATP hydrolysis would prevent the retracting THF from dragging the polypeptide substrate backward.

The single-molecule FRET experiments also indicate that $\mathrm{P}_{\mathrm{i}}$ release is the trigger for clamp opening; in the resulting ADPbound state, the THF is disengaged and polypeptide chain can then slide in either direction ${ }^{19}$. The structural basis for clamp opening is likely the rotation of NBD2 outward and toward SecY ${ }^{13}$, which causes the two NBDs to move apart. This conformational change can be visualized by comparing the positions of NBD1 and NBD2 in crystal structures of B. subtilis SecA obtained in ADP.BeFx and ADP (Supplementary Fig. 7a). The same conformational change occurs in E. coli SecA, but is more pronounced $^{45}$ (Supplementary Fig. $7 \mathrm{~b}$ ). The separation of the NBDs causes the release of the intercalated PPXD loop so that the preceding segment can no longer interact with the substrate ${ }^{19}$ (Supplementary Fig. 7c). 
a

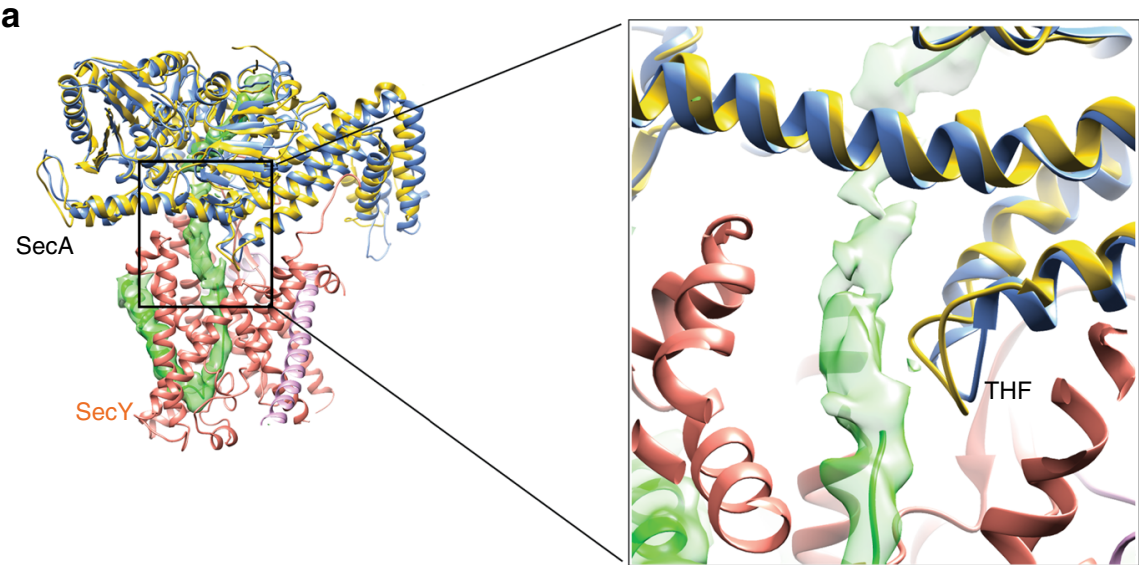

b

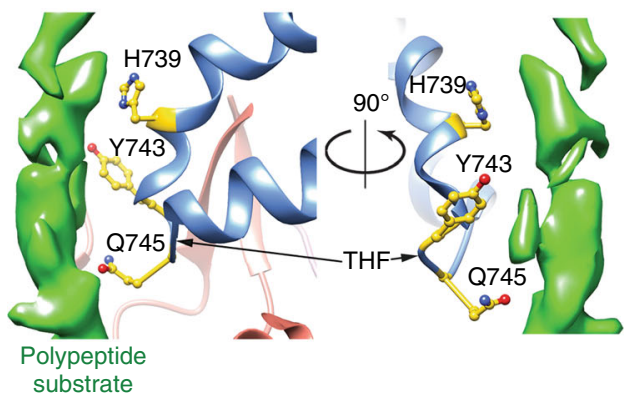

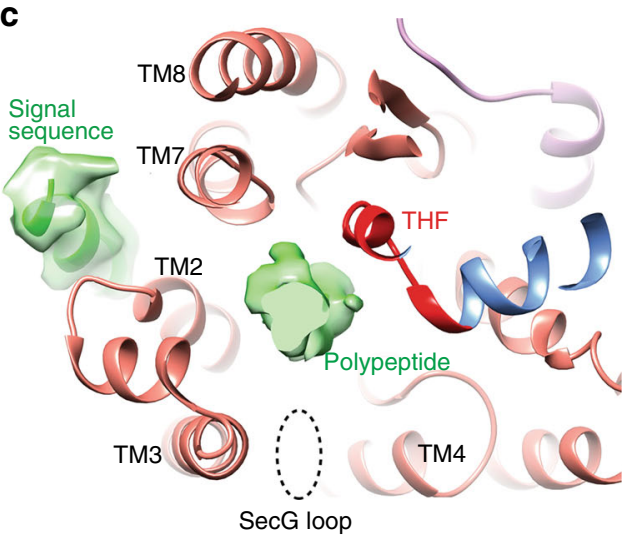

d

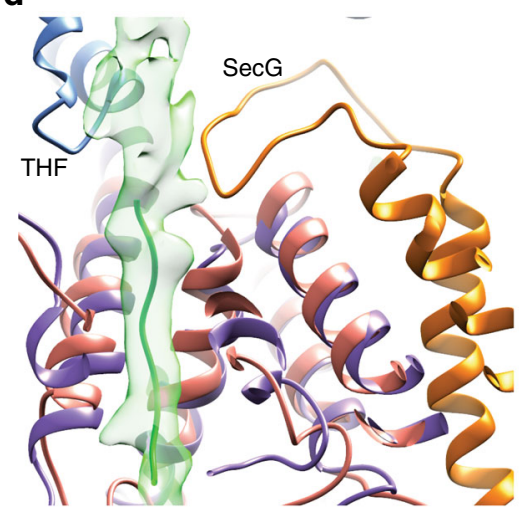

e

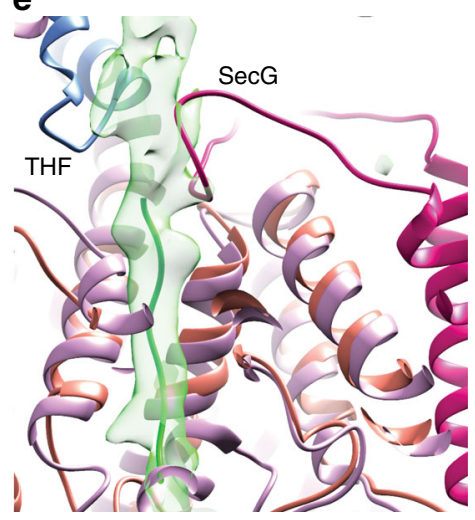

Fig. 5 The polypeptide segment close to the THF. a Superposition of B. subtilis SecA (blue) in the current cryo-EM structure with T. maritima SecA (yellow) in the SecA-SecYEG crystal structure (PDB ID: 3DIN). SecYE and substrate density are from the current structure, while SecYEG and substrate from the crystal structure were deleted. The right panel shows a magnified view of the boxed area. $\mathbf{b}$ Close-up view of THF residues mediating substrate interaction (balls and sticks). c Top view of the polypeptide substrate close to the THF. Density for the substrate is shown in green and the surrounding TMs of SecY in ribbon representation. SecG, which is absent from the present structure, would be located at the position indicated by a dashed oval. $\mathbf{d}$ Superposition of $G$. thermodenitrificans SecYE in the current cryo-EM structure of the active channel with T. maritima SecYEG in a crystal structure of the SecA-primed channel (PDB ID: 3DIN). G. thermodenitrificans SecY is in salmon, T. maritima SecY is in purple, and T. maritima SecG is in gold. e Superposition of G. thermodenitrificans SecYE in the cryo-EM structure of the active channel with T. thermophilus SecYEG in a crystal structure of the idle channel (PDB ID:5AWW). T. thermophilus SecY is in purple and SecG is in magenta. The SecG loop clashes with the polypeptide density

The current structure complements published single-molecule FRET experiments ${ }^{20}$, leading to a coherent model for the coordination of ATP hydrolysis with THF and clamp movements. However, our structure provides only a static snapshot of a single state, and further tests of the translocation models therefore require additional structures of the active translocation complex with different nucleotides and substrates.

\section{Methods}

Protein expression and purification. The expression plasmids, pTet-G. thermodenitrificans $\mathrm{Sec}_{\mathrm{His}} / \mathrm{Y}$ and $\mathrm{pBAD}-\mathrm{proOmpA}-\mathrm{sfGFP}_{3 \mathrm{Cstrep}} /$ B. subtilis SecA778-

Enhancer, were co-transformed into E. coli strain EP51 $1^{33}$ (Supplementary Fig. 1). All residue numberings are for B. subtilis SecA and G. thermodenitrificans SecY and SecE. SecY contained a mutation at residue 60 (G60C). Residues 202-213 in the loop between TMs 5 and 6 of SecY were replaced by the sequence TFGGLN, as in the construct used for crystallization, a mutation that did not affect SecY activity ${ }^{23}$. 
A cysteine was introduced at the 7th residue following the signal sequence of the substrate. The expression of the SecYE was induced by the addition of $200 \mathrm{ng} / \mathrm{mL}$ anhydrotetracycline at OD600 0.8-0.9. The cells were incubated in a shaker for $1.0 \mathrm{~h}$ at $37^{\circ} \mathrm{C}$ and $0.5 \mathrm{~h}$ at $22^{\circ} \mathrm{C}$. Then, $0.15 \% \mathrm{~L}$-arabinose was added to the culture to induce the expression of substrate and SecA at $16^{\circ} \mathrm{C}$ overnight. The cells were harvested and stored at $-80^{\circ} \mathrm{C}$ until use.

The cells were suspended in buffer A $(20 \mathrm{mM}$ Tris- $\mathrm{HCl}$ pH 7.5, $150 \mathrm{mM} \mathrm{NaCl})$ and lysed with an Emulsiflex C3 (Avestin) homogenizer. The membranes were pelleted by ultracentrifugation, washed once with buffer B $(20 \mathrm{mM}$ Tris- $\mathrm{HCl} \mathrm{pH}$ $7.5,300 \mathrm{mM} \mathrm{NaCl}$ ), and solubilized in $0.5 \% \mathrm{n}$-dodecyl $\beta$-D-maltoside (DDM; Anatrace) and $0.5 \%$ lauryl maltose neopentyl glycol (LMNG; Anatrace) in buffer C $(20 \mathrm{mM}$ Tris-HCl pH 7.5, $150 \mathrm{mM} \mathrm{NaCl}, 10 \%$ glycerol). After $1 \mathrm{~h}$ incubation at $4{ }^{\circ} \mathrm{C}$, the solution was clarified by ultracentrifugation. The supernatant was mixed with $5 \mathrm{ml} \mathrm{Ni}$-NTA resin (Thermo Fisher) and incubated for $1 \mathrm{~h}$ at $4{ }^{\circ} \mathrm{C}$. After washing with $15 \mathrm{ml}$ of buffer D (as buffer C, but with $0.02 \% \mathrm{LMNG}$ ) containing $10 \mathrm{mM}$ imidazole and $5 \mathrm{ml}$ of buffer D containing $15 \mathrm{mM}$ imidazole, the protein was eluted with $5.5 \mathrm{ml}$ of buffer D containing $250 \mathrm{mM}$ imidazole. The eluted material was immediately loaded onto a column pre-packed with $0.75 \mathrm{ml}$ StrepTactin resin (IBA). The protein was eluted with buffer C containing $0.1 \%$ digitonin (Biosynth) and $10 \mathrm{mM}$ desthiobiotin. About $1.5 \mathrm{ml}$ of the eluent was collected and immediately supplemented with $5 \mathrm{mM} \mathrm{MgCl}$ and $1 \mathrm{mM}$ ADP.BeFx. The protein was concentrated with an Amicon filter (100 kD MWCO, EMD Millipore) and loaded onto a Superdex 200 10/300 column (GE Healthcare) in buffer G (20 mM HEPES-NaOH pH 7.0, $100 \mathrm{mM} \mathrm{NaCl}, 0.1 \%$ digitonin, $5 \mathrm{mM}$ $\mathrm{MgCl}_{2}, 1 \mathrm{mM}$ ADP.BeFx). The peak fractions were concentrated to $\sim 6 \mathrm{mg} / \mathrm{ml}$, aliquoted, and flash-frozen in liquid nitrogen. The protein was stored at $-80^{\circ} \mathrm{C}$.

The plasmid encoding an anti-SecY Nanobody with an N-terminal His-SUMO tag was transformed into E. coli strain BL21 (DE3). The cells were grown in LB medium at $37^{\circ} \mathrm{C}$. Protein expression was induced with $0.5 \mathrm{mM} \mathrm{IPTG}$ at $\mathrm{OD}_{600}=$ 0.6. The expression was continued at $20^{\circ} \mathrm{C}$ overnight. About $7 \mathrm{~g}$ of cells were obtained from $1 \mathrm{~L}$ of culture. The cells were lysed by sonication. After centrifugation, the supernatant was mixed with $4 \mathrm{ml}$ of Ni-NTA resin (Thermo Fisher) and incubated at $4{ }^{\circ} \mathrm{C}$ for $1 \mathrm{~h}$. The protein was eluted with $250 \mathrm{mM}$ imidazole, and then diluted and concentrated in buffer A with $1 \mathrm{mM}$ dithiothreitol (DTT) to remove imidazole. The SUMO tag was cut off by incubating with the SUMO protease at $4{ }^{\circ} \mathrm{C}$ overnight. The sample was then applied to a Ni-NTA resin again to remove the SUMO tag. The nanobody was further purified by gel filtration on a HiLoad 16/600 Superdex $75 \mathrm{pg}$ column in buffer A. The purified nanobody was concentrated to $30 \mathrm{mg} / \mathrm{ml}$.

The MSP1D1 protein was purified according to the published protocols ${ }^{46}$. Briefly, the His-tagged MSP1D1 was expressed in E. coli strain BL21 (DE3). The cells from $1 \mathrm{~L}$ culture were lysed by sonication in the lysis buffer $(40 \mathrm{mM}$ Tris $\mathrm{pH}$ $8.0,300 \mathrm{mM} \mathrm{NaCl}, 10 \%$ glycerol, $1 \%$ Triton-X100). The protein was purified on a Ni-NTA resin, followed by gel filtration. Finally, MSP1D1 was concentrated to $11 \mathrm{mg} / \mathrm{ml}$ in gel filtration buffer $(20 \mathrm{mM}$ Tris $\mathrm{pH} 7.4,150 \mathrm{mM} \mathrm{NaCl})$.

Improving nanobody affinity by yeast display. GeneMorph II Random Mutagenesis Kit (Agilent) was used to introduce random mutations into the DNA sequence of $\mathrm{AYC} 08$, the nanobody used for determining the previous crystal structure $^{23}$. The mutation rate was estimated to $\mathrm{be} \sim 6 \mathrm{bp} / 1 \mathrm{~kb}$. The pool of mutated DNA sequences was co-electroporated with a linearized yeast display vector, pYDS649HM ${ }^{47}$, into Saccharomyces cerevisiae (strain: BJ5465). The display of the nanobody mutants on the yeast cell surface was induced by galactose. It was estimated that the sequence diversity of the mutant library was about $10^{8}$. The induced cells were stained with an anti-HA antibody labeled with Alexa647 (BioLegend) and SecA-OAIns-sfGFP/SecYE ${ }^{23}$. The stained cells were sorted and analyzed by flow cytometry. Nanobodies with the highest SecY affinity were enriched after several rounds of sorting. Several mutations were identified and sequenced.

\section{Reconstitution of the translocation complex into nanodiscs. The purified} translocation complex was mixed with MSP1D1 and E. coli polar lipids (Avanti Lipids, $40 \mathrm{mg} / \mathrm{ml}$ dissolved in $0.5 \%$ DDM) at a molar ratio of 1:2:25. Bio-beads SM2 (Bio-Rad) were then added to the mixture and incubated at $4{ }^{\circ} \mathrm{C}$ overnight to remove the detergents. The complex was further purified on a StrepTactin resin to remove empty nanodiscs. The reconstituted and purified nanodiscs had a concentration of $1.6 \mathrm{mg} / \mathrm{ml}$ in the elution buffer $(20 \mathrm{mM}$ HEPES pH 7.0, $150 \mathrm{mM}$ $\mathrm{NaCl}, 10 \mathrm{mM}$ desthiobiotin, $5 \mathrm{mM} \mathrm{MgCl}, 1 \mathrm{mM} \mathrm{BeFx}$, and $0.5 \mathrm{mM}$ ADP.

Cryo-EM sample preparation and data collection. The freshly prepared nanodisc samples were mixed with the anti-SecY nanobody at a molar ratio of 1:1.2 before vitrification. Holey-carbon gold grids (Quantifoil, R1.2/1.3) were glow-discharged with a plasma cleaner, and for each grid $5 \mu \mathrm{l}$ sample was used. Cryo-grid preparation was performed with an FEI Vitrobot Mark IV with the inner chamber set at $4{ }^{\circ} \mathrm{C}$ and $100 \%$ humidity. The cryo-grids were screened with a $200 \mathrm{kV}$ FEI Talos Arctica microscope (FEI Ceta camera). Data sets were collected on a $300 \mathrm{kV}$ FEI Titan Krios TEM (Gatan K2 summit camera) with GIF Quantum energy filter (Gatan). The images were collected at a dose rate of $4.8 \mathrm{e}^{-} / \mathrm{s} / \AA^{2}$ with an exposure time of $12 \mathrm{~s}$. Movie stacks (40 frames each) were recorded with the software serial $\mathrm{EM}^{48}$ under low-dose conditions. The magnification was set at $\times 130,000$ and the defocus ranged from -1.5 to $-2.5 \mu \mathrm{m}$. Statistics for data collection was summarized in Supplementary Table 1.

Image processing. A total of 5968 movie stacks were collected. Motion correction and electron-dose weighting were performed by using MotionCor ${ }^{49}$. The program $\mathrm{Gctf}^{50}$ was used to estimate the contrast transfer function (CTF) parameters. Images of high quality were selected for further image processing on the basis of the CTF power spectra of the corrected images. A small set of 2093 particles were hand-picked and subjected to $2 \mathrm{D}$ classification using RELION3.0 ${ }^{51}$. Class average images of high quality were selected and used as templates for particle auto-picking with RELION3.0. After two rounds of 2D classification (Supplementary Fig. 2), 472,724 particles (two batches of data) were selected for 3D classification. The initial three-dimensional (3D) model was calculated using cisTEM ${ }^{52}$. For the first batch of 290,961 particles, a cascade of 3D classification with a binning factor of two was applied to further exclude bad particles (Supplementary Fig. 2c). Among the eight groups from the first round of 3D classification, two with good secondary structure features were combined (108,684 particles) for a second round of 3D classification. Based on the map appearance and the resolution of secondary features, three classes (56,857 particles) were kept for 3D refinement. The second batch of data (181,763 particles) was similarly processed. A final set of 130,153 particles from both data batches were combined for refinement that resulted in maps of resolutions of 3.88 and $3.50 \AA$ after mask-based post-processing. The particles were subjected to further CTF and $3 \mathrm{D}$ refinement. The final resolution is $3.82 \AA$ without post-processing and $3.45 \AA$ after mask-based post-processing (Supplementary Figs $2 \mathrm{~d}$ and e). All the resolution estimations were based on goldstandard Fourier Shell Correlation (FSC) 0.143 criteria. We also tried to classify the particles using mask-based classification focusing on regions of the peptide substrate. However, only marginal improvement was achieved.

Models for SecA-OAIns/SecYE/AYC08 (PDB ID 5EUL) and GFP-enhancer (PDB ID $3 \mathrm{~K} 1 \mathrm{~K}$ ) were fit into the electron density map. The polypeptide substrate, the plug, SecE, the helical wing domain of SecA, and some regions at the SecA-SecY interfaces were rebuilt. The model was refined in real space using Phenix ${ }^{53}$. Model validation was done with MolProbity ${ }^{54}$.

\section{Data availability}

The cryo-EM maps have been deposited in the Electron Microscopy Data Bank under accession numbers EMD-9731. The atomic structure coordinates have been deposited in the Protein Data Bank under the accession number 6ITC. All other data can be obtained from the corresponding author upon reasonable request.

Received: 1 April 2019 Accepted: 10 June 2019

Published online: 28 June 2019

\section{References}

1. Lycklama, A. N. J. A. \& Driessen, A. J. The bacterial Sec-translocase: structure and mechanism. Philos Trans. R. Soc. Lond. B Biol. Sci. 367, 1016-1028 (2012).

2. Denks, K. et al. The Sec translocon mediated protein transport in prokaryotes and eukaryotes. Mol. Membr. Biol. 31, 58-84 (2014).

3. Collinson, I., Corey, R. A. \& Allen, W. J. Channel crossing: how are proteins shipped across the bacterial plasma membrane? Philos. Trans. R. Soc. Lond. B Biol. Sci. 370, 20150025 (2015).

4. Voorhees, R. M. \& Hegde, R. S. Toward a structural understanding of cotranslational protein translocation. Curr. Opin. Cell Biol. 41, 91-99 (2016).

5. Rapoport, T. A., Li, L. \& Park, E. Structural and mechanistic insights into protein translocation. Annu Rev. Cell Dev. Biol. 33, 369-390 (2017).

6. Tsirigotaki, A., De Geyter, J., Sostaric, N., Economou, A. \& Karamanou, S Protein export through the bacterial Sec pathway. Nat. Rev. Microbiol 15, 21-36 (2017)

7. Nishiyama, K., Mizushima, S. \& Tokuda, H. A novel membrane protein involved in protein translocation across the cytoplasmic membrane of Escherichia coli. EMBO J. 12, 3409-3415 (1993).

8. Egea, P. F. \& Stroud, R. M. Lateral opening of a translocon upon entry of protein suggests the mechanism of insertion into membranes. Proc. Natl Acad. Sci. USA 107, 17182-17187 (2010).

9. Van den Berg, B. et al. X-ray structure of a protein-conducting channel. Nature 427, 36-44 (2004).

10. Tanaka, Y. et al. Crystal structures of SecYEG in lipidic cubic phase elucidate a precise resting and a peptide-bound state. Cell Rep. 13, 1561-1568 (2015).

11. Tsukazaki, T. et al. Conformational transition of Sec machinery inferred from bacterial SecYE structures. Nature 455, 988-991 (2008).

12. Matlack, K. E., Misselwitz, B., Plath, K. \& Rapoport, T. A. BiP acts as a molecular ratchet during posttranslational transport of prepro-alpha factor across the ER membrane. Cell 97, 553-564 (1999).

13. Hunt, J. F. et al. Nucleotide control of interdomain interactions in the conformational reaction cycle of SecA. Science 297, 2018-2026 (2002). 
14. Zimmer, J., Nam, Y. S. \& Rapoport, T. A. Structure of a complex of the ATPase SecA and the protein-translocation channel. Nature 455, 936-U32 (2008).

15. Erlandson, K. J. et al. A role for the two-helix finger of the SecA ATPase in protein translocation. Nature 455, 984-987 (2008).

16. Bauer, B. W. \& Rapoport, T. A. Mapping polypeptide interactions of the SecA ATPase during translocation. Proc. Natl Acad. Sci. USA 106, 20800-20805 (2009).

17. Allen, W. J. et al. Two-way communication between SecY and SecA suggests a Brownian ratchet mechanism for protein translocation. Elife 5, e15598 (2016).

18. Corey, R. A. et al. ATP-induced asymmetric pre-protein folding as a driver of protein translocation through the Sec machinery. Elife 8, e41803 (2019).

19. Bauer, B. W., Shemesh, T., Chen, Y. \& Rapoport, T. A. A. "Push and slide" mechanism allows sequence-insensitive translocation of secretory proteins by the SecA ATPase. Cell 157, 1416-1429 (2014).

20. Catipovic, M. A., Bauer, B. W., Loparo, J. J. \& Rapoport, T. A. Protein translocation by the SecA ATPase occurs by a power-stroke mechanism. EMBO J. 38, e101140 (2019).

21. Economou, A. \& Wickner, W. SecA promotes preprotein translocation by undergoing ATP-driven cycles of membrane insertion and deinsertion. Cell 78, 835-843 (1994)

22. Banerjee, T., Zheng, Z., Abolafia, J., Harper, S. \& Oliver, D. The SecA protein deeply penetrates into the SecYEG channel during insertion, contacting most channel transmembrane helices and periplasmic regions. J. Biol. Chem. 292, 19693-19707 (2017).

23. Li, L. et al. Crystal structure of a substrate-engaged SecY protein-translocation channel. Nature 531, 395-399 (2016).

24. Karamanou, S. et al. Escherichia coli SecA truncated at its termini is functional and dimeric. FEBS Lett. 579, 1267-1271 (2005).

25. Pedelacq, J. D., Cabantous, S., Tran, T., Terwilliger, T. C. \& Waldo, G. S. Engineering and characterization of a superfolder green fluorescent protein. Nat. Biotechnol. 24, 79-88 (2006).

26. Kirchhofer, A. et al. Modulation of protein properties in living cells using nanobodies. Nat. Struct. Mol. Biol. 17, 133-138 (2010).

27. Bayburt, T. H., Grinkova, Y. V. \& Sligar, S. G. Self-assembly of discoidal phospholipid bilayer nanoparticles with membrane scaffold proteins. Nano Lett. 2, 853-856 (2002).

28. Findik, B. T., Smith, V. F. \& Randall, L. L. Penetration into membrane of amino-terminal region of SecA when associated with SecYEG in active complexes. Protein Sci. 27, 681-691 (2018).

29. $\mathrm{Li}, \mathrm{W}$. et al. The plug domain of the SecY protein stabilizes the closed state of the translocation channel and maintains a membrane seal. Mol. Cell 26, 511-521 (2007).

30. Martoglio, B., Hofmann, M. W., Brunner, J. \& Dobberstein, B. The proteinconducting channel in the membrane of the endoplasmic reticulum is open laterally toward the lipid bilayer. Cell 81, 207-214 (1995).

31. Plath, K., Mothes, W., Wilkinson, B. M., Stirling, C. J. \& Rapoport, T. A. Signal sequence recognition in posttranslational protein transport across the yeast ER membrane. Cell 94, 795-807 (1998).

32. McKnight, C. J., Briggs, M. S. \& Gierasch, L. M. Functional and nonfunctional LamB signal sequences can be distinguished by their biophysical properties. J. Biol. Chem. 264, 17293-17297 (1989).

33. Park, E. \& Rapoport, T. A. Preserving the membrane barrier for small molecules during bacterial protein translocation. Nature 473, 239-242 (2011).

34. Chen, Y., Bauer, B. W., Rapoport, T. A. \& Gumbart, J. C. Conformational changes of the clamp of the protein translocation ATPase SecA. J. Mol. Biol. 427, 2348-2359 (2015).

35. Sharma, V. et al. Crystal structure of Mycobacterium tuberculosis SecA, a preprotein translocating ATPase. Proc. Natl Acad. Sci. USA 100, 2243-2248 (2003).

36. Yazdi, A. K., Vezina, G. C. \& Shilton, B. H. An alternate mode of oligomerization for E. coli SecA. Sci. Rep. 7, 11747 (2017).

37. Zimmer, J., Li, W. \& Rapoport, T. A. A novel dimer interface and conformational changes revealed by an X-ray structure of B. subtilis SecA. J. Mol. Biol. 364, 259-265 (2006).

38. Zimmer, J. \& Rapoport, T. A. Conformational flexibility and peptide interaction of the translocation ATPase SecA. J. Mol. Biol. 394, 606-612 (2009).

39. Voorhees, R. M. \& Hegde, R. S. Structure of the Sec61 channel opened by a signal sequence. Science 351, 88-91 (2016).

40. Gogala, M. et al. Structures of the Sec61 complex engaged in nascent peptide translocation or membrane insertion. Nature 506, 107-110 (2014).

41. Bischoff, L., Wickles, S., Berninghausen, O., van der Sluis, E. O. \& Beckmann, R. Visualization of a polytopic membrane protein during SecY-mediated membrane insertion. Nat. Commun. 5, 4103 (2014).

42. Sato, K., Mori, H., Yoshida, M., Tagaya, M. \& Mizushima, S. Short hydrophobic segments in the mature domain of ProOmpA determine its stepwise movement during translocation across the cytoplasmic membrane of Escherichia coli. J. Biol. Chem. 272, 5880-5886 (1997).
43. Nouwen, N., Berrelkamp, G. \& Driessen, A. J. Charged amino acids in a preprotein inhibit SecA-dependent protein translocation. J. Mol. Biol. 386, 1000-1010 (2009)

44. Liang, F. C., Bageshwar, U. K. \& Musser, S. M. Position-dependent effects of polylysine on Sec protein transport. J. Biol. Chem. 287, 12703-12714 (2012)

45. Papanikolau, Y. et al. Structure of dimeric SecA, the Escherichia coli preprotein translocase motor. J. Mol. Biol. 366, 1545-1557 (2007).

46. Denisov, I. G., Grinkova, Y. V., Lazarides, A. A. \& Sligar, S. G. Directed selfassembly of monodisperse phospholipid bilayer Nanodiscs with controlled size. J. Am. Chem. Soc. 126, 3477-3487 (2004).

47. McMahon, C. et al. Yeast surface display platform for rapid discovery of conformationally selective nanobodies. Nat. Struct. Mol. Biol. 25, 289-296 (2018).

48. Mastronarde, D. N. Automated electron microscope tomography using robust prediction of specimen movements. J. Struct. Biol. 152, 36-51 (2005).

49. Zheng, S. Q. et al. MotionCor2: anisotropic correction of beam-induced motion for improved cryo-electron microscopy. Nat. Methods 14, 331-332 (2017).

50. Zhang, K. Gctf: Real-time CTF determination and correction. J. Struct. Biol. 193, 1-12 (2016).

51. Zivanov, J. et al. RELION-3: new tools for automated high-resolution cryo-EM structure determination. ELife 7, e42166 (2018)

52. Grigorieff, N. Frealign: an exploratory tool for single-particle Cryo-EM. Methods Enzym. 579, 191-226 (2016).

53. Afonine, P. V. et al. Real-space refinement in PHENIX for cryo-EM and crystallography. Acta Crystallogr D. Struct. Biol. 74, 531-544 (2018).

54. Chen, V. B. et al. MolProbity: all-atom structure validation for macromolecular crystallography. Acta Crystallogr D. Biol. Crystallogr 66, 12-21 (2010).

\section{Acknowledgements}

We thank Xudong Wu for insightful discussions on protein purification and cryo-EM sample preparation, Andrew Kruse for help with yeast display and display vectors, the Core Facilities at School of Life Sciences Peking University for assistance with negative staining EM, and the Electron Microscopy Laboratory of Peking University and the cryoEM platform of Peking University for help with data collection. The computation was supported by High-performance Computing Platform of Peking University. This work was supported by the Ministry of Science and Technology of China (2016YFA0500700 to N.G.), the National Natural Science Foundation of China (NSFC) (31725007 and 31630087 to N.G.; 31800625 to C.M.; 31870835 to L.L.), and the China Postdoctoral Science Foundation (2018M631249 to C.M.). C.M. is supported by a postdoctoral fellowship from the Peking-Tsinghua Centre for Life Sciences. T.A.R. is supported by NIH grant GM R01GM052586 and is an HHMI investigator.

\section{Author contributions}

C.M. performed cryo-EM sample preparation and structure determination. X.W., D.S. and L.L. prepared the protein samples. E.P. designed the SecA-nanobody fusion and did pilot purifications of the complex. The project was started in the laboratory of T.A.R. L.L. and N.G. supervised the project. L.L., N.G., C.M., M.C. and T.A.R. prepared the paper.

\section{Additional information}

Supplementary Information accompanies this paper at https://doi.org/10.1038/s41467019-10918-2.

Competing interests: The authors declare no competing interests.

Reprints and permission information is available online at http://npg.nature.com/ reprintsandpermissions/

Peer review information: Nature Communications thanks the anonymous reviewer(s) for their contribution to the peer review of this work.

Publisher's note: Springer Nature remains neutral with regard to jurisdictional claims in published maps and institutional affiliations.

Open Access This article is licensed under a Creative Commons Attribution 4.0 International License, which permits use, sharing, adaptation, distribution and reproduction in any medium or format, as long as you give appropriate credit to the original author(s) and the source, provide a link to the Creative Commons license, and indicate if changes were made. The images or other third party material in this article are included in the article's Creative Commons license, unless indicated otherwise in a credit line to the material. If material is not included in the article's Creative Commons license and your intended use is not permitted by statutory regulation or exceeds the permitted use, you will need to obtain permission directly from the copyright holder. To view a copy of this license, visit http://creativecommons.org/ licenses/by/4.0/.

(C) The Author(s) 2019 